\title{
Vidrios y grisallas del s. XV de la Cartuja de Miraflores (Burgos): Caracterización y estado de conservación
}

\author{
N. CARMONA* , M. GARCÍA-HERAS* , C. GIL** Y M.A. VILLEGAS* \\ * Centro Nacional de Investigaciones Metalúrgicas, CENIM-CSIC. \\ Avda. Gregorio del Amo, 8. 28040 Madrid. \\ ** Fundación Centro Nacional del Vidrio. Real Fábrica de Cristales de San Ildefonso. \\ Po Pocillo, 1. 40100 La Granja de San Ildefonso, Segovia.
}

\begin{abstract}
Este trabajo presenta los resultados de la caracterización fisicoquímica y el diagnóstico del estado actual de conservación de una muestra representativa de vidrios y grisallas procedentes de vidrieras flamencas del s. XV del Monasterio de la Cartuja de Miraflores (Burgos). El objetivo principal del estudio consistió en determinar los mecanismos y las causas de los importantes procesos de degradación y alteración observados en las superficies de los vidrios utilizados en estas vidrieras, con el fin de proponer medidas para su conservación preventiva. Las muestras seleccionadas se caracterizaron mediante microscopía óptica (MO), espectroscopía de absorción UV/Vis, fluorescencia de rayos X (FRX), microscopía electrónica de barrido de emisión de campo (MEBEC) y convencional (MEB) y microanálisis de dispersión de energía de rayos X (EDX). La composición química de los vidrios estudiados permite su clasificación como vidrios potásico-cálcicos de transición entre los períodos medieval y renacentista. Los resultados indican que estos vidrios han sufrido una alteración muy severa con una intensa desalcalinización superficial, destrucción de la red estructural vítrea y formación de depósitos insolubles procedentes de los productos de corrosión, que puede atribuirse al efecto sinérgico de los procesos de degradación química y de una actividad biológica.
\end{abstract}

Palabras clave: Vidrio; Grisalla; Vidrieras históricas; Degradación; Conservación.

\section{Glass and grisailles of the 15th century from the Cartuja de Miraflores (Burgos): characterisation and state of conservation}

This paper reports the results derived from a combined physicochemical characterisation and state of conservation diagnosis study undertaken on a representative ensamble of 15th century Flemish stained glasses and grisailles from the Cartuja de Miraflores Monastery (Burgos, Spain). The main goal of the research was to determine the mechanisms and causes of the important degradation and alteration processes observed on the surfaces of these glasses, in order to propose preventive conservation steps. Selected samples were charecterised by optical microscopy (OM), UV/Vis spectrophotometry, X-ray fluorescence (XRF), field emission and conventional scanning electron microscopy (FESEM and SEM) and energy dispersive X-ray microanalysis (EDX). The chemical composition of the glasses studied allows their classification as potassium-lime silicate glasses of the Medieval-Renaissance transitional period. Resulting data indicate that these glasses have experienced a very harsh alteration with a strong superficial dealkalinisation, destruction of the glass structural network and formation of insoluble deposits from corrosion by-products, which can be attributed to the synergic effect of chemical degradation processes and biological activity.

Keywords: Glass; Grisaille; Historical stained glass windows; Damage; Conservation.

\section{INTRODUCCIÓN}

Las vidrieras flamencas del Monasterio de la Cartuja de Miraflores (Burgos) se localizan principalmente en la única nave de su iglesia, aunque existen otras de menores dimensiones en los ventanales del claustro. El Monasterio se fundó en 1442 por Juan II de Castilla y su construcción se finalizó en 1488 durante el reinado de Isabel la Católica, siendo uno de los conjuntos góticos más destacados de fines del s. XV. Fue precisamente la reina Isabel la Católica la que por encargo expreso ordenó importar en 1484, directamente de Flandes, las vidrieras de la Cartuja de Miraflores, lo que sin duda constituye un testimonio fidedigno de la importancia que tuvieron las relaciones comerciales y artísticas durante esta época entre los Países Bajos y Burgos (1).

El conjunto de vidrieras ubicado en la iglesia está formado por grandes composiciones, que ocupan todo el ventanal, dedicadas a la pasión, vida y gloria de Cristo. Estas vidrieras se realizaron con un gran dominio de la técnica, una representación minuciosa del detalle y abundantes juegos cromáticos en los que destaca el empleo constante del amarillo de plata (2). Según parecen indicar las firmas descubiertas en tres de los ventanales, las vidrieras pudieron haber sido elaboradas por el maestro vidriero Nicolás Romboust, uno de los vidrieros flamencos más destacados del s. XV.

La caracterización fisicoquímica y el diagnóstico del estado actual de conservación de los vidrios y de las grisallas, pinturas vitrificables coloreadas con iones de metales de transición, empleados en las vidrieras históricas, genera un tipo de información indispensable y determinante en la toma de decisiones sobre el tipo de restauración que requieren y sobre las medidas que deben adoptarse para su preservación futura. En este artículo se presentan los resultados de un trabajo de estas características, realizado a partir de una muestra representativa de vidrios y grisallas procedentes de cinco de los ventanales del lado norte de la iglesia 
del Monasterio de la Cartuja de Miraflores, en los que se detectaron signos evidentes de una corrosión muy avanzada. El objetivo principal del estudio consistió, por tanto, en determinar los mecanismos y las causas de los importantes procesos de degradación y alteración que han sufrido estos materiales a lo largo del tiempo, como consecuencia de sus propias características y de las condiciones ambientales a las que han estado expuestos, con el fin de conocer su estado actual de conservación y los factores que pueden influir de forma negativa para su conservación en el futuro.

\section{PARTE EXPERIMENTAL}

\subsection{Muestras estudiadas}

Para la realización de este estudio se seleccionaron un total de nueve muestras representativas de vidrios de distinta coloración: incoloro, incoloro con una fina capa de amarillo de plata en la cara externa, azul en masa, violeta en masa,

\section{Caras externas}
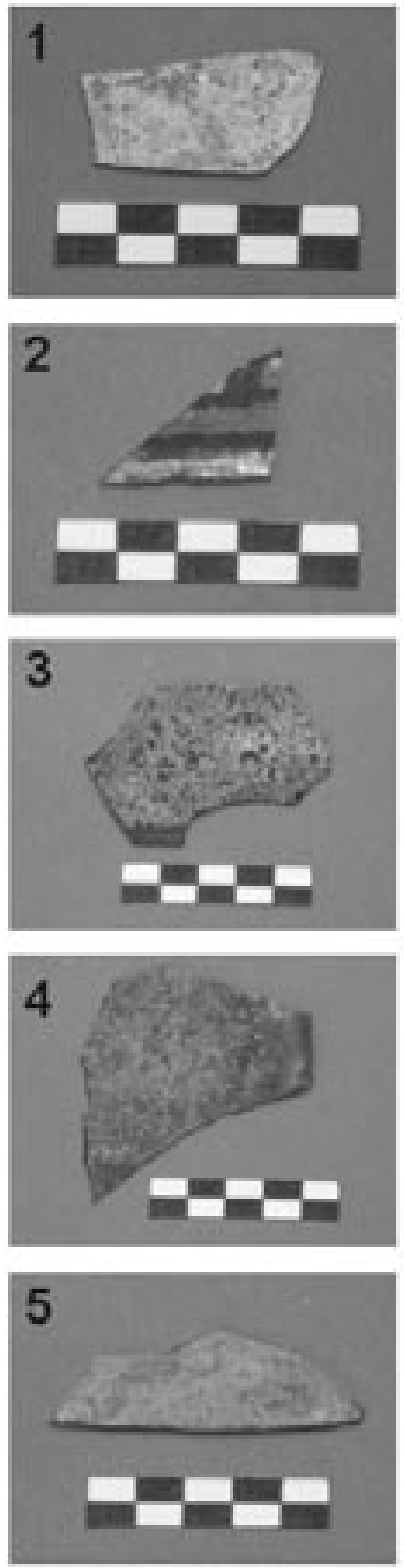

Caras internas
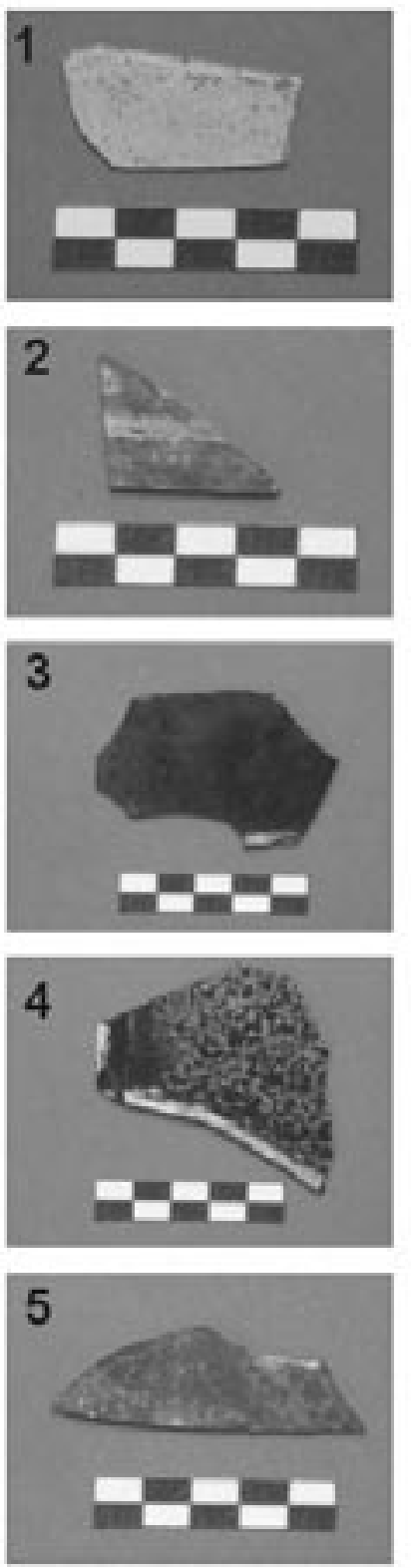

TABLA I. NOMENCLATURA Y ASPECTO DE LAS MUESTRAS ESTUDIADAS.

\begin{tabular}{|c|c|c|c|}
\hline \multirow{2}{*}{ Nomenclatura } & \multicolumn{2}{|c|}{ Coloración vidrio } & \multirow{2}{*}{ Grisalla } \\
\hline & En masa & Superficial & \\
\hline M-1 & incoloro & --- & no \\
\hline M-2 & incoloro & $\begin{array}{c}\text { amarillo de } \\
\text { plata } \\
\text { (cara externa) }\end{array}$ & $\begin{array}{c}\mathrm{si} \\
\text { (cara interna) }\end{array}$ \\
\hline M-3 & azul & --- & no \\
\hline M-4 & azul & --- & no \\
\hline M-5 & incoloro & --- & $\begin{array}{c}\mathrm{si} \\
\text { (cara interna) }\end{array}$ \\
\hline M-6 & violeta & --- & no \\
\hline M-7 & incoloro-verdoso & $\begin{array}{l}\text { rojo plaqué } \\
\text { (cara interna) }\end{array}$ & no \\
\hline M-8 & incoloro-verdoso & $\begin{array}{c}\text { rojo plaqué } \\
\text { (cara interna) }\end{array}$ & no \\
\hline M-9 & verde & --- & no \\
\hline
\end{tabular}

\section{Caras externas}
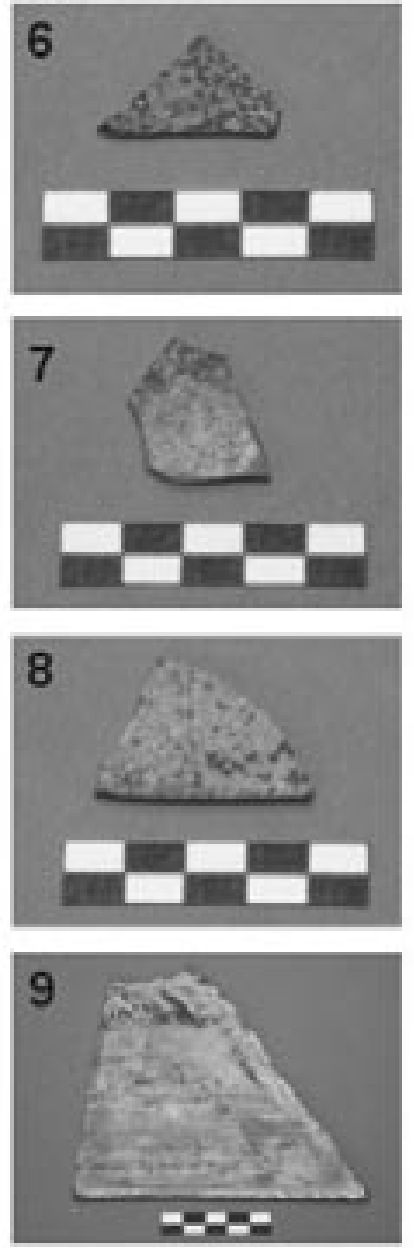

\section{Caras internas}
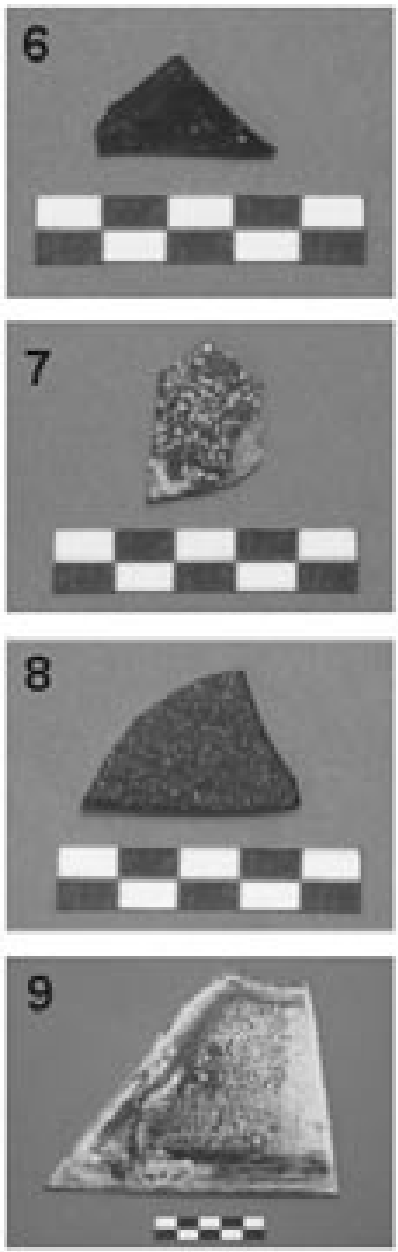

Fig. 1.- Fotografías de las nueve muestras de vidrio seleccionadas y analizadas tal como se recibieron en el laboratorio. 
incoloro-verdoso con una fina capa de rojo plaqué o vidrio doblado en la cara interna y verde en masa (Tabla I). En dos de estas muestras se pudieron también estudiar sus grisallas que se localizaban en la cara interna y que presentaban un color marrón oscuro. Las muestras M-1 a M-8 se correspondían con fragmentos desubicados en los que pudieron llevarse a cabo análisis destructivos o parcialmente destructivos, mientras que en la muestra M-9 sólo pudo determinarse su espectro de absorción visible puesto que debía reponerse en su ubicación original en el panel correspondiente. En las fotografías agrupadas en la Figura 1 puede observarse el aspecto general que presentaban las muestras seleccionadas en las que, sobre todo en las caras externas, eran claramente visibles signos de una corrosión muy avanzada.

\subsection{Técnicas de análisis utilizadas}

La caracterización fisicoquímica y el diagnóstico del estado actual de conservación de las muestras se realizó utilizando las siguientes técnicas complementarias: microscopía óptica (MO), espectroscopía de absorción UV/Vis, fluorescencia de rayos $\mathrm{X}$ (FRX), microscopía electrónica de barrido de emisión de campo (MEBEC) y convencional (MEB) y microanálisis de dispersión de energía de rayos X (EDX).

La morfología macroscópica de las alteraciones superficiales se estudió mediante observaciones de MO de ambas caras de cada muestra con un microscopio de luz reflejada Olympus Mod. DP-11. El estudio de los cromóforos (especies químicas responsables de la coloración de los vidrios), se llevó a cabo mediante espectroscopía de absorción UV/Vis con un espectrofotómetro Perkin Elmer Mod. Lambda 19, una vez eliminadas por pulido manual las costras superficiales de corrosión que presentaban todas las muestras. Este análisis se efectuó con el fin de corroborar si el color que mostraban los vidrios de visu, pese a las importantes costras de corrosión, era el que correspondía al cromóforo presente en los mismos. El análisis químico semicuantitativo mediante FRX sólo se realizó en aquellas muestras más representativas (M-1, M3, M-5 y M-7), tomando porciones de vidrio exento de productos de corrosión y cualquier otro tipo de depósitos. Cuando fue necesario, éstos fueron eliminados por pulido manual. Asimismo, la fina capa de rojo plaqué o vidrio doblado de la cara interna de la muestra M-7, también fue eliminada por pulido con el fin de obtener la composición del vidrio base incoloro-verdoso sin interferencias de la capa roja de vidrio doblado. Para los análisis de FRX se utilizó un espectrómetro Philips Mod. PW-1404 con tubo de rodio. Las muestras se prepararon en polvo que fue prensado posteriormente en pastillas de ácido bórico, utilizando una mezcla de n-butilmetacrilato y acetona (10:90\% en peso) como aglomerante. Las observaciones microestructurales de ambas caras de cada muestra se realizaron mediante MEBEC con las superficies recubiertas con carbono como medio conductor. El equipo utilizado fue un microscopio Jeol Mod. JSM 6500F. Los microanálisis de EDX se efectuaron con un espectrómetro Pentafet Link acoplado al microscopio mencionado. Por último, las observaciones microestructurales de las dos muestras con grisalla se realizaron en secciones transversales pulidas, con la muestra recubierta con oro depositado utilizando un equipo Sputter Coater Cressington Mod. 108 Auto. En dichas observaciones el equipo empleado fue un microscopio electrónico de barrido convencional Philips XL-30 Mod. DX4, con espectrómetro EDX de la misma marca comercial.

\section{RESULTADOS Y DISCUSIÓN}

\subsection{Análisis morfológico}

Todas las muestras presentaron signos de una alteración superficial profunda. En general, las caras externas de los fragmentos de vidrio (orientadas hacia fuera del edificio) mostraron un estado de conservación mucho más deficiente que las correspondientes caras internas (orientadas hacia dentro del edificio).

La morfología superficial de los vidrios, que en condiciones normales de conservación debería aparecer prácticamente lisa, presentó numerosas alteraciones en forma de costras fuertemente adheridas, cuyo color y extensión es variable dependiendo de la muestra. En las caras externas de los vidrios se apreció la formación de abundantes costras heterogéneas de color marrón oscuro muy interconectadas que, prácticamente, cubrían toda la superficie del vidrio (Figura 2a, 2b). También se observaron cráteres y picaduras (Figura 2a, zona superior), a menudo igualmente interconectadas, rellenas de depósitos de aspecto similar a las costras. En algunas muestras, como en el caso del vidrio incoloro $\mathrm{M}-5$, las costras presentaron un color más blanquecino (Figura 2c); mientras que en otras, como en el caso del vidrio incoloro-verdoso M-7 (Figura 2d), las costras eran mucho más oscuras y compactas. El aspecto de estas costras indica que pueden estar formadas por una acumulación de sales insolubles procedentes de los productos de corrosión del vidrio subyacente (3).
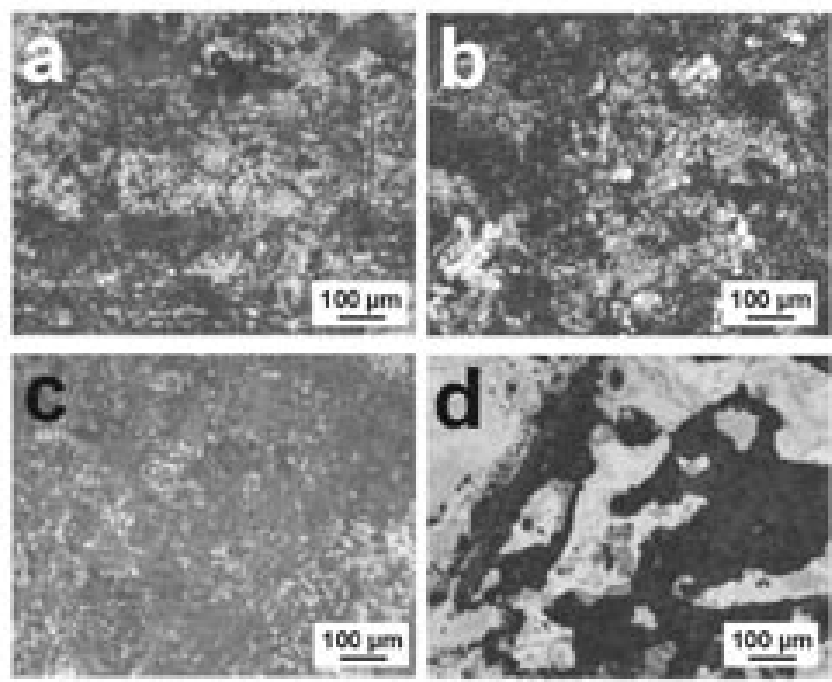

Fig. 2.- Imágenes de MO de algunos de los vidrios estudiados. Caras externas. (a) Muestra M-2. (b) Muestra M-3. (c) Muestra M-5. (d) Muestra M-7.

Las caras internas de los vidrios presentaron un mejor estado de conservación. En muchos casos, sólo se apreciaron pequeñas picaduras y una escasa acumulación de depósitos de corrosión (Figura 3a, 3c). No obstante, algunas muestras presentaron signos de una mayor alteración superficial, en las que se observó la aparición de capas finas irisadas (Figura $3 b$ ), a veces cubriendo casi toda la superficie (Figura 3d), que pueden atribuirse a la formación de capas de gel de sílice. Dichas capas se originan tras un proceso de desalcalinización de la superficie de los vidrios cuando se han sometido a un porcentaje elevado de humedad relativa en condiciones 


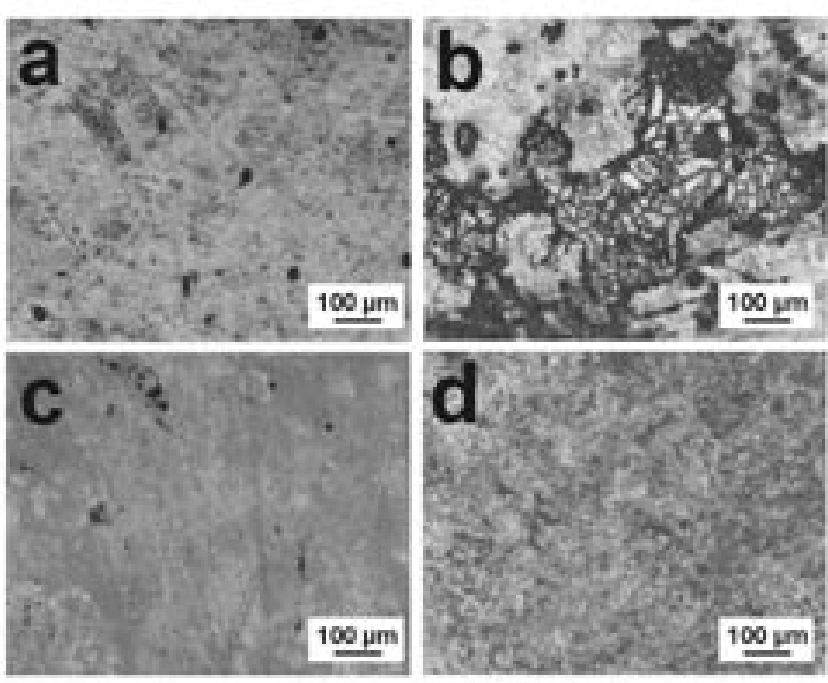

Fig. 3.- Imágenes de MO de algunos de los vidrios estudiados. Caras internas. (a) Muestra M-2. (b) Muestra M-3. Capa irisada. (c) Muestra M-4. (d) Muestra M-7. Capa irisada.

estacionarias, como el que tiene lugar en el interior de los edificios cuando las condensaciones de vapor de agua son frecuentes y prolongadas (4).

\subsection{Análisis de los cromóforos}

La Figura 4 muestra los espectros de absorción UV/Vis de cada uno de los cromóforos determinados. Los espectros se han ordenado siguiendo la numeración de las muestras, ofreciendo únicamente un espectro de aquellos vidrios que presentaron igual coloración. En primer lugar, las muestras M-1 y M-5, de aspecto incoloro enmascarado con una densa
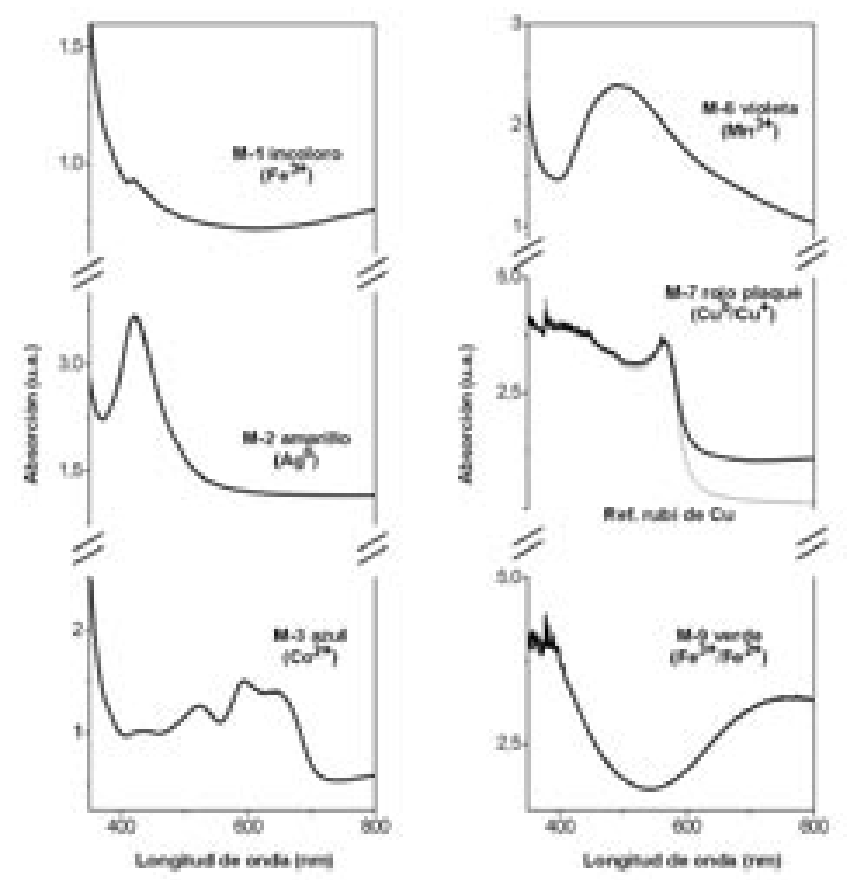

Fig. 4.-Espectros de absorción visible obtenidos en algunas de las muestras de vidrio analizadas. La línea de puntos se refiere a un vidrio rubí de cobre utilizado como referencia. costra de corrosión, mostraron una débil coloración amarilla. El correspondiente espectro de absorción visible presentó una banda de intensidad débil a $420 \mathrm{~nm}$ que se asigna, tanto por su forma como por su intensidad, a iones $\mathrm{Fe}^{3+}$ responsables de esta ligera tonalidad amarilla que se observa visualmente. En la muestra $\mathrm{M}-2$, constituida por un vidrio incoloro con una fina capa amarilla en su cara externa, se confirmó la absorción debida a la presencia de plata en forma de coloides metálicos de tamaño nanométrico. Su espectro de absorción visible presentó una banda muy intensa a $440 \mathrm{~nm}$, correspondiente a la resonancia de los plasmones superficiales de los coloides de $\mathrm{Ag}^{0}$. La incorporación de la plata se realizó probablemente por un procedimiento de cementación en el que los iones $\mathrm{Ag}^{+}$se intercambian con los iones alcalinos del vidrio incoloro base. Posteriormente, los iones $\mathrm{Ag}^{+}$se reducen térmicamente $\mathrm{a} \mathrm{Ag}^{0}$ y se agregan dando lugar a los coloides responsables de la intensa coloración amarilla (5).

Las muestras M-3 y M-4 presentaron tres bandas intensas de absorción en su espectro visible a 540, 590 y $640 \mathrm{~nm}$ respectivamente, atribuibles a la presencia de iones $\mathrm{Co}^{2+}$. Dichos iones son los responsables del intenso color azul de estos vidrios (6). La muestra M-6 debe su tonalidad violeta a la presencia de iones $\mathrm{Mn}^{3+}$, que fue confirmada en el correspondiente espectro de absorción por la presencia de una banda muy intensa a 499 nm (7). Las muestras M-7 y M-8 están constituidas por un vidrio incoloro, de tonalidad residual verdosa, con una fina capa de rojo plaqué o vidrio doblado en la cara interna. Sus espectros visibles presentaron una banda de absorción muy intensa a $565 \mathrm{~nm}$ atribuible a iones $\mathrm{Cu}^{+} \mathrm{y}$ átomos de $\mathrm{Cu}^{0}$, formando coloides de tamaño nanométrico responsables de la coloración roja rubí que se observa (5). Los espectros obtenidos se compararon con un espectro de referencia de un vidrio rojo rubí actual (Figura 4, línea de puntos), con el fin de comprobar la correcta identificación de este cromóforo.

Por último, la muestra M-9 presentó un espectro de absorción visible complejo, formado por la superposición de varias bandas intensas cuyos máximos se sitúan, por un lado, alrededor de $400 \mathrm{~nm} \mathrm{y}$, por otro lado, entre $630 \mathrm{y}$ $800 \mathrm{~nm}$ atribuibles a la presencia de iones $\mathrm{Fe}^{2+}$ y $\mathrm{Fe}^{3+}$. $\mathrm{La}$ coloración azul que producirían los iones $\mathrm{Fe}^{2+}$ se debe a la banda de 1050-1100 nm, cuya gran anchura hace que parte de ella penetre en el espectro visible en la región del rojo (Figura 4, banda ancha entre 630 y $800 \mathrm{~nm}$ ). Las absorciones observadas para esta muestra no podrían asignarse a las de los iones $\mathrm{Cr}^{3+}$ que deberían aparecer mejor resueltas. Además, la presencia de iones $\mathrm{Cr}^{3+}$ únicamente podría explicarse como impurezas, lo cual es poco probable pues en la fecha en la que se manufacturaron estos vidrios los compuestos de cromo eran desconocidos y no se utilizaban como colorantes. La coloración azul que proporcionan los iones $\mathrm{Fe}^{2+}$ y la coloración amarilla de los iones $\mathrm{Fe}^{3+}$, dan lugar a la coloración verde que se aprecia visualmente $(7,8)$.

\subsection{Composición química de los vidrios}

En la Tabla II se recogen los resultados de los análisis químicos semicuantitativos mediante FRX de las muestras más representativas. Aunque en todas ellas se eliminaron mediante pulido manual las capas superficiales de alteración y de productos de corrosión, en estos resultados no debe descartarse un posible efecto de meteorización de las capas más profundas, ya que las muestras presentaron un deterioro muy severo. 
TABLA II. RESULTADOS DEL ANÁLISIS QUÍMICO SEMICUANTITATIVO DE ALGUNOS DE LOS VIDRIOS ESTUDIADOS, MEDIANTE FRX

\begin{tabular}{|c|c|c|c|c|c|c|c|c|c|c|c|}
\hline \multirow{2}{*}{ Muestra } & \multicolumn{11}{|c|}{ Óxidos (\% en peso) } \\
\hline & $\mathrm{Na}_{2} \mathrm{O}$ & $\mathrm{MgO}$ & $\mathrm{Al}_{2} \mathrm{O}_{3}$ & $\mathrm{SiO}_{2}$ & $\mathrm{P}_{2} \mathrm{O}_{5}$ & $\mathrm{~K}_{2} \mathrm{O}$ & $\mathrm{CaO}$ & $\mathrm{MnO}$ & $\mathrm{Fe}_{2} \mathrm{O}_{3}$ & $\mathrm{CoO}$ & Otros \\
\hline $\begin{array}{c}\mathrm{M}-1 \\
\text { incoloro }\end{array}$ & 2,85 & 3,72 & 1,37 & 59,10 & 1,15 & 4,57 & 23,52 & 1,07 & 0,50 & - & 2,15 \\
\hline $\begin{array}{l}\text { M-3 } \\
\text { azul }\end{array}$ & 2,13 & 3,50 & 1,72 & 56,17 & 1,24 & 5,33 & 25,01 & 1,04 & 1,01 & 0,15 & 2,70 \\
\hline $\begin{array}{c}\mathrm{M}-5 \\
\text { incoloro }\end{array}$ & 1,33 & 3,13 & 2,70 & 52,65 & 1,24 & 7,25 & 26,72 & 2,55 & 0,50 & - & 1,93 \\
\hline $\begin{array}{c}\text { M-7 } \\
\text { incoloro- } \\
\text { verdoso }\end{array}$ & 3,26 & 4,40 & 1,83 & 55,93 & 1,25 & 5,00 & 25,00 & 0,70 & 0,78 & - & 1,85 \\
\hline
\end{tabular}

En todos los casos el contenido de $\mathrm{SiO}_{2}$ se encuentra entre el 52 y el $59 \%$ en peso, lo que en principio indica que se trata de vidrios de estabilidad media o vidrios de tipo 5 según la clasificación de Müller y col. (9). Estos vidrios son propios del período de transición entre la Baja Edad Media y el Renacimiento. Asimismo, atendiendo a su contenido de sílice, también podrían clasificarse como vidrios potásicos estables según la denominación de Bettenbourg (10). Los contenidos en $\mathrm{Na}_{2} \mathrm{O}$ varían entre 1 y $3 \%$ en peso, mientras que los de $\mathrm{K}_{2} \mathrm{O}$ lo hacen entre 4 y $7 \%$ en peso. Estos intervalos de concentración de óxidos alcalinos concuerdan con las dos clasificaciones anteriores $(9,10)$. Los porcentajes de $\mathrm{CaO}$ oscilan entre 23 y $27 \%$ en peso, lo que está de acuerdo con la clasificación de Bettenbourg (10) pero no con la de Müller y col. (9), ya que en los vidrios de tipo 5 el porcentaje de $\mathrm{CaO}$ no sobrepasa el 20 $\%$ en peso.

En los vidrios estudiados, el $\mathrm{P}_{2} \mathrm{O}_{5}$ está presente en concentraciones de alrededor de un $1,2 \%$ en peso, porcentaje algo menor del señalado en los vidrios de tipo 5, que se sitúa entre el 3-10\% en peso (9). Las concentraciones de $\mathrm{MgO}$ varían entre 3 y $4 \%$ en peso. El papel que desempeña el $\mathrm{MgO}$ en la estructura de los vidrios es semejante al del $\mathrm{CaO}$, ya que también actúa como un óxido modificador/estabilizador de la red vítrea (7). Desde este punto de vista, el contenido de $\mathrm{MgO}$ podría asimilarse al de $\mathrm{CaO}$, en cuyo caso el porcentaje global de óxidos estabilizadores se situaría en el intervalo 26$31 \%$ en peso. Este intervalo sobrepasa el señalado para el $\mathrm{CaO}$ en el vidrio de tipo 5 de la clasificación de Müller y col. (9). No obstante, los vidrios estudiados podrían encajar bien en esta clasificación, ya que aún presentan mayores divergencias en los contenidos de otros óxidos, con respecto a los otros tipos de vidrio propuestos por dichos autores. Se trata, por tanto, de vidrios de transición entre el período medieval y el renacentista, en los que la concentración de $\mathrm{K}_{2} \mathrm{O}$ se va reduciendo y la de $\mathrm{Na}_{2} \mathrm{O}$ se va aumentando progresivamente. Por otro lado, el contenido de $\mathrm{Al}_{2} \mathrm{O}_{3}$ en los vidrios estudiados se sitúa entre 1 y $3 \%$ en peso. La incorporación de este óxido aumenta la estabilidad de la red vítrea. Por ello, el porcentaje de óxidos formadores de vidrio debe contemplar la suma de los contenidos de $\mathrm{SiO}_{2}$ y $\mathrm{Al}_{2} \mathrm{O}_{3}$, que en el presente caso se eleva alrededor de un $60 \%$ en peso, lo que concuerda igualmente con el tipo 5 de la clasificación de Müller y col. (9).

El resto de componentes minoritarios $\left(\mathrm{MnO}, \mathrm{Fe}_{2} \mathrm{O}_{3}\right.$ y $\mathrm{CoO})$ son óxidos que se comportan como cromóforos de los vidrios, bien añadidos intencionadamente ( $\mathrm{MnO}$ y $\mathrm{CoO})$ o incorporados con las materias primas como impurezas $\left(\mathrm{Fe}_{2} \mathrm{O}_{3}\right)$. Los porcentajes de óxido de manganeso varían entre 0,7 y 2,6 $\%$ en peso, mientras que los de $\mathrm{Fe}_{2} \mathrm{O}_{3}$ lo hacen entre 0,5 y $1,0 \%$ en peso. Los contenidos de óxido de manganeso determinados mediante FRX concuerdan bien con los cromóforos asignados mediante espectroscopía UV/Vis (Figura 4). En cualquier caso, las concentraciones de óxido de manganeso parecen muy elevadas, lo que hace pensar que posiblemente fuera añadido como decolorante o "jabón de vidriero" (3). La muestra M-6 de color violeta, cuyo análisis por FRX no pudo realizarse por disponer de una cantidad insuficiente, debe su coloración a la presencia de manganeso en estado de oxidación III (Figura 4). Esto podría indicar que los vidrieros flamencos del s. XV distinguían entre "vidrios incoloros" (con tonalidad amarilla leve conteniendo óxido de manganeso como decolorante de las impurezas de óxido de hierro) y "vidrios violetas" (con mayor proporción de iones $\mathrm{Mn}^{3+} \mathrm{y}$ tonalidad violeta intensa).

Los contenidos de $\mathrm{Fe}_{2} \mathrm{O}_{3}$ concuerdan bien con los porcentajes esperados de dichas impurezas en las arenas aportadoras de sílice que se utilizaron. Por otro lado, el CoO sólo se detectó, como era de esperar, en la muestra de vidrio azul M-3 en un porcentaje habitual para su uso como cromóforo azul. En la Tabla II se han incluido como otros componentes los óxidos de cobre (responsable de la coloración rojo rubí de las muestras de vidrio plaqué o doblado M-7 y M-8), estroncio, cinc, bario, etc. En general pueden ser considerados como óxidos aportados por las impurezas de las materias primas utilizadas en la fabricación de estos vidrios, excepto en el caso del cobre, cuya adición fue sin duda intencionada para proporcionar la coloración roja.

\subsection{Observaciones y microanálisis superficiales mediante MEBEC-EDX}

Las superficies externas de los vidrios analizados mostraron abundantes picaduras y cráteres de tamaño macroscópico frecuentemente interconectados, de profundidad variable pero a menudo suficiente para haber provocado el desprendimiento de pequeños fragmentos de vidrio en forma de estratos o escamas, de las que quedan partículas de aspecto terroso y pulverulento. En las zonas en las que aún se conservaba alguna textura lisa o casi lisa, propia de lo que pudiera ser el vidrio original inalterado, aparecían numerosas grietas y picaduras de menor tamaño, a menudo cubiertas de restos de depósitos procedentes, probablemente, de los productos de corrosión del vidrio (Figura 5a, 5b). La muestra M-3 presentó, en la zona de textura lisa menos deteriorada (Figura 5a, zona 1, Tabla III), una disminución del contenido de óxidos alcalinos y alcalinotérreos $\left(\mathrm{Na}_{2} \mathrm{O}, \mathrm{K}_{2} \mathrm{O}, \mathrm{CaO}\right)$ y un aumento relativo de los óxidos formadores de red $\left(\mathrm{SiO}_{2}, \mathrm{Al}_{2} \mathrm{O}_{3}\right)$, respecto a la composición inicial del vidrio (Tabla II). Esto indica que se ha producido una desalcalinización superficial, con lo que dicha superficie se ha enriquecido proporcionalmente 

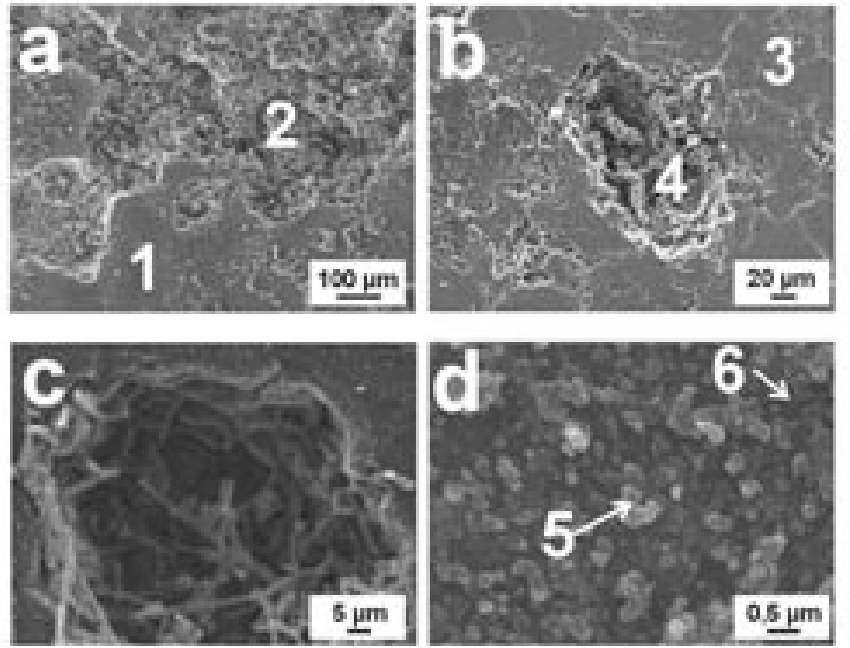

Fig. 5.-Micrografías de MEBEC de algunos de los vidrios estudiados. Caras externas. (a) Muestra M-3. (b) Muestra M-6. (c) Muestra M-7. (d) Muestra M-3.

en óxidos formadores que han dado lugar a una capa de gel de sílice. En la zona de picaduras más deteriorada (Figura 5a, zona 2, Tabla III), el fenómeno que puede identificarse microestructuras filamentosas del interior de los cráteres y picaduras, podría atribuirse a depósitos originados no sólo por la corrosión química del vidrio, que no suele producir este tipo de microestructuras, sino también por el efecto sinérgico de una degradación de tipo biológico relacionada posiblemente con la actividad metabólica de hongos y otros microorganismos $(12,13)$. Finalmente, la Figura 5d muestra el aspecto terroso y pulverulento de un fragmento de vidrio desprendido en el interior de uno de los cráteres observados en la muestra M-3. Los microanálisis mediante EDX realizados en distintas zonas (Figura 5d, zonas 5 y 6, Tabla III) demostraron que se había producido una disminución importante de $\mathrm{CaO}$, algo menor de $\mathrm{K}_{2} \mathrm{O}$ y $\mathrm{MgO}$, así como un aumento considerable de $\mathrm{SiO}_{2}$ y $\mathrm{P}_{2} \mathrm{O}_{5}$.

Las superficies internas de los vidrios analizados, como ya se había observado en el análisis morfológico mediante $\mathrm{MO}$, presentaron menores signos de alteración. No obstante, junto a zonas oscuras de textura más o menos lisa correspondientes al vidrio original poco alterado, aparecieron otras áreas con picaduras y cráteres, a veces interconectados (Figura 6a). Al observar estas picaduras a mayores aumentos (Figura 6b), se pudo comprobar que también se hallaban rellenas de depósitos de aspecto blanquecino y que la superficie del vidrio en sus alrededores aparecía frecuentemente descamada con un aspecto terroso y pulverulento, como el observado en

TABLA III. RESULTADOS DE LOS MICROANÁLISIS REALIZADOS EN LA SUPERFICIE DE ALGUNOS DE LOS VIDRIOS ESTUDIADOS, MEDIANTE EDX

\begin{tabular}{|c|c|c|c|c|c|c|c|c|c|c|c|c|}
\hline \multirow{2}{*}{ Muestra } & \multirow{2}{*}{ Zona } & \multicolumn{11}{|c|}{ Óxidos (\% en peso) } \\
\hline & & $\mathrm{Na}_{2} \mathrm{O}$ & $\mathrm{MgO}$ & $\mathrm{Al}_{2} \mathrm{O}_{3}$ & $\mathrm{SiO}_{2}$ & $\mathrm{P}_{2} \mathrm{O}_{5}$ & $\mathrm{SO}_{2}$ & $\mathrm{Cl}^{-}$ & $\mathrm{K}_{2} \mathrm{O}$ & $\mathrm{CaO}$ & $\mathrm{MnO}$ & $\mathrm{Fe}_{2} \mathrm{O}_{3}$ \\
\hline \multirow{2}{*}{$\begin{array}{c}\text { M-3 } \\
\text { (Fig. 5a) }\end{array}$} & 1 & 1,8 & 3,1 & 2,0 & 63,0 & 3,0 & 0,5 & 0,4 & 4,2 & 20,3 & 0,6 & 1,0 \\
\hline & 2 & - & 1,0 & 1,9 & 43,3 & 5,2 & 16,1 & - & 1,1 & 30,1 & 1,3 & - \\
\hline $\begin{array}{c}\text { M-6 } \\
\text { (Fig. 5b) }\end{array}$ & 3 & - & 0,8 & 2,6 & 88,7 & 1,2 & - & 1,8 & 0,9 & 2,4 & 0,6 & 1,0 \\
\hline \multirow{2}{*}{$\begin{array}{c}\text { M-3 } \\
\text { (Fig. 5d) }\end{array}$} & 5 & 2,0 & 2,5 & 1,9 & 65,2 & 3,3 & 1,4 & 0,7 & 4,3 & 17,1 & 0,6 & 1,0 \\
\hline & 6 & 2,1 & 2,5 & 2,0 & 64,9 & 3,0 & 1,3 & 0,7 & 4,5 & 17,3 & 0,6 & 1,1 \\
\hline \multirow{2}{*}{$\begin{array}{c}\text { M-4 } \\
\text { (Fig. 6c) }\end{array}$} & 7 & 1,8 & 3,3 & 1,8 & 60,5 & 3,5 & - & 0,4 & 5,0 & 21,3 & 0,9 & 1,5 \\
\hline & 8 & 1,3 & 1,6 & 3,9 & 69,8 & 2,6 & 2,8 & 1,0 & 3,6 & 11,7 & - & 1,7 \\
\hline
\end{tabular}

es el mismo pero más acentuado, de modo que no sólo se ha producido una desalcalinización superficial y formación de capa de gel de sílice, sino que también se ha perdido masa del vidrio, apareciendo nuevos componentes (azufre) e incrementándose el porcentaje de $\mathrm{CaO}$, como consecuencia de la acumulación de depósitos de sales insolubles procedentes de los productos de corrosión (11). Aunque no se poseen datos comparativos de la composición inicial del vidrio al no contar con cantidad suficiente de muestra para realizar el análisis por FRX, el fragmento violeta M-6 también parece presentar, en la zona de textura más o menos lisa, una disminución de óxidos alcalinos y alcalinotérreos y un aumento relativo de los óxidos formadores de red (Figura 5b, zona 3, Tabla III). Sin embargo, en el interior de los cráteres, cuyas microestructuras presentaron un aspecto filamentoso más claro, aparte del fenómeno de desalcalinización y formación de la capa de gel de sílice, se detectó un porcentaje elevado de cloro y algo menor de azufre (Figura 5b, zona 4, Tabla III). En la Figura 5c se ofrece una micrografía a mayores aumentos del aspecto de estas microestructuras filamentosas observadas en el interior de los cráteres, en este caso de la muestra M-7. La presencia de concentraciones elevadas de azufre, cloro y calcio en las
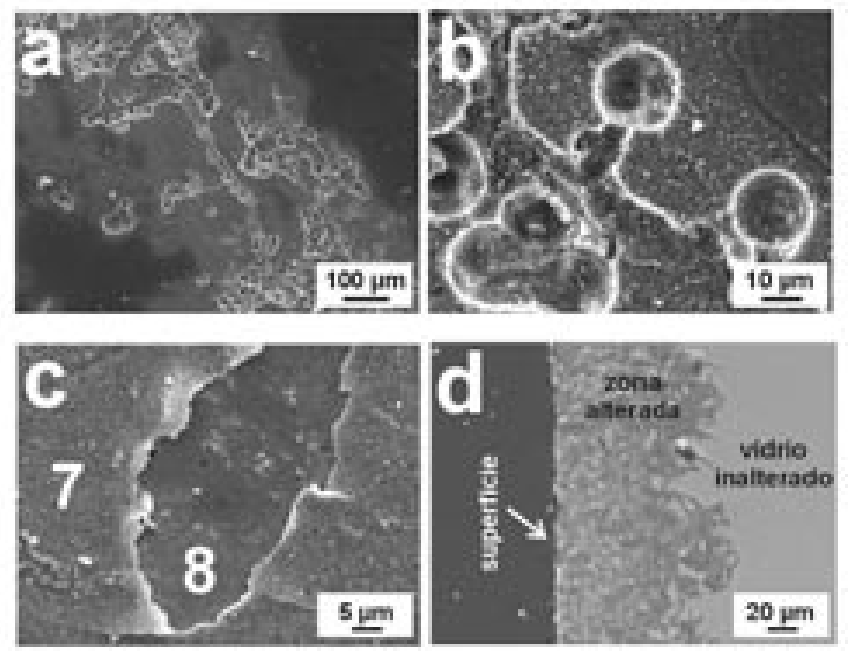

Fig. 6.- Micrografías de MEBEC de algunos de los vidrios estudiados. (a) Muestra M-3. Cara interna. (b) Muestra M-3. Cara interna. (c) Muestra M-4. Cara interna. (d) Muestra M-2. Sección transversal pulida de la superficie interna en una zona sin grisalla. 
el interior de los cráteres de las superficies externas (Figura 5d). En la Figura 6c se muestra un detalle de una de las zonas descamadas y desprendidas en la superficie del vidrio M-4. En la zona más externa de aspecto terroso y pulverulento (Figura 6c, zona 7, Tabla III), el microanálisis EDX determinó un enriquecimiento en $\mathrm{SiO}_{2}$ y $\mathrm{P}_{2} \mathrm{O}_{5^{\prime}}$ y un decrecimiento del contenido de $\mathrm{CaO}$, respecto a la composición inicial que presentaba un vidrio similar, también de coloración azul, como el de la muestra M-3 (Tabla II). Por el contrario, la zona descamada interna de aspecto más oscuro y textura más lisa (Figura 6c, zona 8, Tabla III), mostró un mayor incremento de $\mathrm{SiO}_{2}, \mathrm{Al}_{2} \mathrm{O}_{3}, \mathrm{SO}_{2}$ y Cl , y un decrecimiento más pronunciado de los óxidos alcalinos y alcalinotérreos. Esto indica que se ha producido, al igual que en las superficies externas, una desalcalinización superficial con formación de una capa de gel de sílice. En las superficies internas, no obstante, no se apreció una acumulación tan importante de depósitos de sales insolubles procedentes de los productos de corrosión del vidrio, debido a que, en líneas generales, los procesos de alteración han sido menos intensos. De todas formas, las alteraciones de las superficies internas no deben menospreciarse ya que, en algunas muestras, la observación pormenorizada en sección transversal pulida demostró que éstas habían penetrado en el cuerpo del vidrio hasta una profundidad de unos $100 \mu \mathrm{m}$ (Figura 6d).

Las observaciones y microanálisis superficiales mediante MEBEC-EDX permiten sostener que los importantes procesos de degradación y alteración sufridos por los vidrios de las vidrieras flamencas del s. XV de la Cartuja de Miraflores, se han producido como consecuencia de un intenso ataque hidrolítico y el efecto de otros agentes externos de meteorización como la contaminación medioambiental $(3,4)$. Los cráteres y las picaduras pueden conectarse con las gotas de agua de condensación que provocan el ataque hidrolítico del vidrio y la formación de una fina capa de gel de sílice. A su vez, si estas gotas contienen disueltas especies ácidas como $\mathrm{SO}_{2}, \mathrm{CO}_{2}$ o $\mathrm{NO}_{x}$ procedentes de la contaminación medioambiental, se produce un ataque ácido de la superficie del vidrio que causa su desalcalinización. La extracción de iones de potasio y, sobre todo, de iones de calcio origina la formación de depósitos insolubles procedentes de los productos de corrosión del vidrio. Finalmente, si las condiciones ambientales permanecen estáticas, la presencia de especies alcalinas sobre la superficie del vidrio puede incrementar el $\mathrm{pH}$ circundante hasta valores básicos, produciéndose entonces un ataque de tipo alcalino que provoca la despolimerización de la red vítrea y la pérdida de masa del propio vidrio. Una última consecuencia de la desalcalinización y la formación de depósitos insolubles puede ser que éstos sean colonizados por hongos y otros microorganismos capaces de metabolizar estos compuestos $(12,13)$, produciéndose así un efecto sinérgico de degradación química y actividad biológica en la superficie del vidrio.
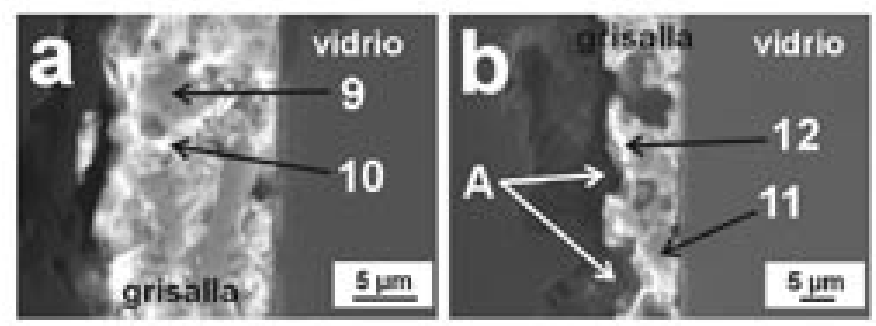

Fig. 7.-Micrografías de MEB de algunas de las grisallas estudiadas. Secciones transversales pulidas de las superficies internas. (a) Muestra M-2. (b) Muestra M-5.

\subsection{Análisis de las grisallas}

La grisalla de la muestra M-2 presentó una superficie irregular de aspecto rugoso y deteriorado (Figura 7a, zona izda). El cuerpo de la grisalla estaba constituido por granos de textura homogénea y aspecto oscuro, cuyo microanálisis mediante EDX indicó la presencia mayoritaria de $\mathrm{Fe}_{2} \mathrm{O}_{3}$ y $\mathrm{PbO}$, así como $\mathrm{SiO}_{2}$ en menor proporción (Figura 7a, zona 9, Tabla IV). Estos granos se hallaban inmersos en una matriz de aspecto heterogéneo y más clara, compuesta fundamentalmente por $\mathrm{PbO}, \mathrm{Fe}_{2} \mathrm{O}_{3}, \mathrm{SiO}_{2}$ y $\mathrm{K}_{2} \mathrm{O}$, junto a otros óxidos minoritarios (Figura 7a, zona 10, Tabla IV). En su conjunto se trata, por tanto, de una grisalla constituida por una mezcla de granos de óxido de hierro embebidos en una matriz vitrificable compuesta principalmente de $\mathrm{PbO}$ y $\mathrm{SiO}_{2}$ y de otros óxidos fundentes como $\mathrm{K}_{2} \mathrm{O}, \mathrm{CuO}, \mathrm{CaO}$, etc.

En la grisalla de la muestra M-5 se observó una superficie extremadamente degradada en la que aparecían grandes oquedades de forma irregular y profundidad considerable, que en algunos casos habían reducido el espesor de la grisalla hasta casi la mitad (Figura $7 \mathrm{~b}$, zonas A). De modo semejante a la muestra $\mathrm{M}-2$, en el cuerpo de esta grisalla también se distinguieron granos de textura homogénea y aspecto oscuro, compuestos mayoritariamente en este caso por $\mathrm{Fe}_{2} \mathrm{O}_{3}$ y $\mathrm{SiO}_{2}$ en una proporción mucho menor, además de otros componentes minoritarios como $\mathrm{Al}_{2} \mathrm{O}_{3}$ y diversos óxidos alcalinos y alcalinotérreos (Figura $7 \mathrm{~b}$, zona 11, Tabla IV). Por otro lado, la matriz presentaba una composición a base de $\mathrm{PbO}, \mathrm{SiO}_{2}, \mathrm{Fe}_{2} \mathrm{O}_{3}$ y $\mathrm{CuO}$ como óxidos mayoritarios de una mezcla vitrificable, $\mathrm{y}$ otros minoritarios como óxidos fundentes $\left(\mathrm{Na}_{2} \mathrm{O}, \mathrm{K}_{2} \mathrm{O}\right.$, $\mathrm{CaO}$, etc.). Por lo demás, en ambas muestras se observó una interfase de unión grisalla/vidrio base poco nítida que indica que la grisalla quedó bien adherida al vidrio mediante un buen proceso de densificación térmica (14).

TABLA IV. RESULTADOS DE LOS MICROANÁLISIS REALIZADOS EN ALGUNAS DE LAS GRISALLAS ESTUDIADAS, MEDIANTE EDX

\begin{tabular}{|c|c|c|c|c|c|c|c|c|c|c|c|c|c|}
\hline \multirow{2}{*}{ Muestra } & \multirow{2}{*}{ Zona } & \multicolumn{12}{|c|}{ Óxidos (\% en peso) } \\
\hline & & $\mathrm{Na}_{2} \mathrm{O}$ & $\mathrm{MgO}$ & $\mathrm{Al}_{2} \mathrm{O}_{3}$ & $\mathrm{SiO}_{2}$ & $\mathrm{P}_{2} \mathrm{O}_{5}$ & $\mathrm{SO}_{2}$ & $\mathrm{PbO}$ & $\mathrm{K}_{2} \mathrm{O}$ & $\mathrm{CaO}$ & $\mathrm{MnO}$ & $\mathrm{Fe}_{2} \mathrm{O}_{3}$ & $\mathrm{CuO}$ \\
\hline \multirow{2}{*}{$\begin{array}{c}\mathrm{M}-2 \\
\text { (Fig. 7a) }\end{array}$} & 9 & - & - & - & 6,0 & 2,4 & - & 6,2 & 1,4 & 1,2 & - & 82,8 & - \\
\hline & 10 & - & - & - & 24,8 & 0,3 & - & 39,3 & 4,2 & 0,7 & - & 28,7 & 2,0 \\
\hline \multirow{2}{*}{$\begin{array}{c}\text { M-5 } \\
\text { (Fig. 7b) }\end{array}$} & 11 & 0,7 & 0,7 & 1,3 & 8,7 & - & 0,9 & - & 0,5 & 0,5 & 0,4 & 86,3 & - \\
\hline & 12 & 1,9 & 0,9 & 1,0 & 29,3 & - & - & 37,4 & 4,1 & 1,8 & 0,4 & 13,3 & 9,9 \\
\hline
\end{tabular}




\section{CONCLUSIONES}

Los vidrios analizados, procedentes de cinco paneles de vidrieras flamencas del s. XV de la Cartuja de Miraflores (Burgos), han presentado una degradación muy severa que puede atribuirse al efecto sinérgico de procesos de alteración química y biológica. Las superficies externas de los fragmentos de vidrio muestran un mayor grado de alteración que las superficies internas, con presencia de picaduras y cráteres de gran tamaño, frecuentemente interconectados y muy profundos. El aspecto de la microestructura de estas picaduras es pulverulento, heterogéneo y, en muchas zonas, filamentoso. Esto indica que los vidrios han sufrido una corrosión química con una intensa desalcalinización superficial, destrucción de la red estructural vítrea y formación de depósitos insolubles procedentes de los productos de corrosión. Además, la microestructura filamentosa observada en la mayor parte de estas picaduras, puede deberse al resultado de una degradación de tipo biológico, en la que hongos y otros microorganismos metabolizan los óxidos del vidrio y sus productos de corrosión, y depositan sobre el propio vidrio degradado diversos productos procedentes de su actividad metabólica. Asimismo, se ha comprobado la coherencia de los resultados obtenidos mediante espectroscopía UV/Vis y análisis químicos mediante FRX en cuanto a la caracterización de los distintos cromóforos de las muestras de vidrio estudiadas.

El análisis de las grisallas ha demostrado que éstas están constituidas por granos de óxidos de hierro embebidos en una matriz vitrificable compuesta mayoritariamente por $\mathrm{PbO}$ y $\mathrm{SiO}_{2}$, además de los correspondientes óxidos fundentes. Respecto a su estado de conservación, se ha observado que sus superficies están extremadamente degradadas, con grandes oquedades de forma irregular y profundidad considerable. Frecuentemente el espesor de la capa de grisalla aparece reducido hasta casi la mitad, debido a la intensa degradación y pérdida de masa que se ha producido.

Ante el avanzado estado de degradación que presentan los vidrios estudiados, lo cual dificulta extraordinariamente la lectura iconográfica de los distintos paneles, las medidas que se proponen para su correcta conservación preventiva son la instalación de un sistema de acristalamientos isotérmicos que aisle, a las vidrieras afectadas, del contacto directo con agentes de meteorización tales como la humedad, las condensaciones, el agua de lluvia y los contaminates químicos y biológicos. En los últimos años, este sistema ha resultado ser el más efectivo para preservar in situ las vidrieras históricas, no sólo de los agentes de meteorización, sino también de los efectos de degradación que el inevitable paso del tiempo provoca sobre cada uno de sus materiales.

\section{AGRADECIMIENTOS}

Este trabajo ha sido parcialmente financiado por el proyecto MCyT MAT2002-02549. Los autores agradecen el apoyo institucional recibido por la Red Temática del CSIC de Patrimonio Histórico y Cultural. El Dr. García-Heras agradece la concesión de un contrato postdoctoral I3P (CSIC-FSE). Asimismo, la Dra. Carmona agradece la concesión de un contrato postdoctoral Marie Curie de la CE.

A nuestro buen colega y amigo, D. Andrés Velasco Pilar, con motivo de su sexagésimo quinto aniversario.

\section{BIBLIOGRAFÍA}

1. J.Ma Azcárate. Arte Gótico en España. Ediciones Cátedra, Madrid, 2000.

2. V. Nieto Alcaide. "Vidrieras", pp. 385-422 en Historia del Arte en Castilla y León, t. III, Arte Gótico. Ediciones Ámbito, Valladolid, 1995.

3. J.M ${ }^{a}$ Fernández Navarro. "Causas del deterioro químico y físico de los vidrios", pp. 17-37 en Jornadas Nacionales sobre Restauración y Conservación de Vidrios, La Granja de San Ildefonso 1999. Eds. J.M Fernández Navarro y P. Pastor Rey de Viñas, Fundación Centro Nacional del Vidrio, La Granja de San Ildefonso (Segovia), 2000.

4. M. García-Heras, C. Gil, N. Carmona y M.A. Villegas. "Efectos de la meteorización sobre los materiales de las vidrieras históricas". Mater. Construcc. 53, 21-34, 2003.

5. J.M ${ }^{a}$ Fernández Navarro y A. La Iglesia. "Estudio de la coloración roja y amarilla de vidrios de la Catedral de Toledo". Bol. Soc. Esp. Ceram. V. 33 333-336, 1994.

6. C.R. Bamford. Colour generation and control in glass. Elsevier Science Publishers, Amsterdam, 1977.

7. J.M ${ }^{a}$ Fernández Navarro. El vidrio. CSIC, Madrid, 2003, $3^{\text {a }}$ edición.

8. T. Bates y R.W. Douglas. "Absorption bands of $\mathrm{Cr}^{3+}$ ions in solutions, crystals, and glasses". J. Soc. Glass Technol. 43, 289-307, 1959.

9. W. Müller, M. Torge y K. Adam. "Ratio of $\mathrm{CaO} / \mathrm{K}_{2} \mathrm{O}>2$ as evidence of a special Renish type of Medieval stained glass". Glass Sci. Technol. 67, 4548, 1994.

10. J.M. Bettenbourg. "Composition et altération des verres de vitraux anciens". Verres Réfrac. 30, 36-42, 1976.

11. W. Müller. "Corrosion phenomena of Medieval stained glasses". Bol. Soc. Esp. Ceram. V. 31-C (1), 219-239, 1992.

12. E. Müller, U. Drewello, R. Drewello, R. Weissmann y S. Wuertz. "In situ analysis of biofilms on historic window glass using confocal laser scanning microscopy". J. Cultural Heritage 2 (1), 31-42, 2001.

13. N. Valentín. "El biodeterioro del vidrio. Métodos de control", pp. 39-46 en Jornadas Nacionales sobre Restauración y Conservación de Vidrios, La Granja de San Ildefonso 1999. Eds. J.M ${ }^{a}$ Fernández Navarro y P. Pastor Rey de Viñas, Fundación Centro Nacional del Vidrio, La Granja de San Ildefonso (Segovia), 2000.

14. N. Carmona. Estudio de los procesos de alteración de vidrieras históricas y de los tratamientos para su restauración y protección. Tesis Doctoral, Univ. de Valladolid, Valladolid, 2002.

Recibido: 12.01 .05

Aceptado: 28.03.05 